\title{
Contraste de la frecuencia de salida del hogar ante la pandemia por COVID-19
}

\author{
Egas Andrea ${ }^{1}$, Santander Carmen Elena ${ }^{2}$, Salazar Marcelo ${ }^{3}$ y Grijalva Alejandro ${ }^{4}$ \\ \{andrea.egas.lopez ${ }^{1}$, carmen.santander ${ }^{2}$, marcelo.salazar.roldan ${ }^{3}$, alejandro.grijalva ${ }^{4}$ \} udla.edu.ec \\ https://orcid.org/0000-0003-2472-9958 ${ }^{1}$, https://orcid.org/0000-0002-5001-3817², \\ https://orcid.org/0000-0003-3673-13263, https://orcid.org/0000-0001-8447-98024 \\ Universidad de las Américas \\ Quito-Ecuador
}

Recibido (13/07/20), Aceptado (28/07/20)

\begin{abstract}
Resumen: En el siguiente estudio se evalúa un panorama con respecto al comportamiento sociológico en un preámbulo tanto antes durante y después de esta crisis social que se está viviendo debido a la pandemia de hoy en día. Por ello para sustentar dicha investigación se realizó un censo en el cuál, mediante el uso de herramientas estadísticas, se pudo realizar una comparación entre un antes y un durante de la pandemia, lo cual facilitaría intrínsicamente a la predicción de una denominada post pandemia. En este texto se pretende detallar el proceso y forma de la encuesta, al igual que los resultados obtenidos de la misma. Se proveen, además, posibles explicaciones para estos resultados, guiándose por las restricciones de salud nacionales como posibles razones para el cambio de comportamiento actual.
\end{abstract}

Palabras Clave: Coronavirus, Pandemia, Frecuencia de salida.

\section{Contrast of the frequency of departure from the home in the face of the COVID-19 pandemic}

\begin{abstract}
The following study contrasts the social behavior before, during and after the forced social isolation due to the pandemic. With the use of statistical tools, a census was made to compare the habits of a population and their changes, with the objective of identifying patterns for a prediction of future events after the health crisis. This text details the methodology, process, and results of the census and several possible explanations to these outcomes, considering the national health measures and regulations as reasons to the change in social behavior.
\end{abstract}

Keywords: Coronavirus, Pandemic, Output frequency. 


\section{I.INTRODUCCIÓN}

Actualmente estamos pasando por una pandemia la cual ha afectado a todo el mundo. Esta enfermedad logró fragilizar la mayoría de los países en el mundo en todo sentido, ya que las personas hoy en día tienen un comportamiento diferente cuando tienen contacto con la sociedad. Para poder regresar a la normalidad, primero se tendrá que encontrar la cura a este virus mortal, lo importante es que las personas se queden en casa para tratar de evitar el contagio. Esta cuarenta que hemos pasado, nos ha llevado a buscar nuevas formas de vivir, como, por ejemplo: jugar en familia, arreglar cosas que siempre postergamos, hacer ejercicio, ha unido a familias que estaban separadas y con todo esto llegar a tener una buena convivencia. Nuestro estudio tiene el objetivo de ver cómo la gente ha cambiado sus hábitos antes y después de la pandemia, ya que hoy en día la gente ya respeta las filas, el espacio personal, más cuidados higiénicos, en fin, existe mucha más educación que antes. Toda acción conlleva a una reacción, esto hace lógico que la situación actual de salud este condicionada a posibles irregularidades en la conservación del ambiente, en la forma de alimentación, en las condiciones de vida, entre otras.

Para empezar, podemos ver muchos cambios en el comportamiento general de las personas; no solo entre familia, sino también en todo tipo de relación social. Una de las ventajas que existe en esta pandemia es que la tecnología ha ayudado a estar en contacto con nuestros seres queridos, las visitas que eran frecuentes se han cambiado a llamadas virtuales; no solo hablando de la familia sino también de esas amistades que uno está acostumbrado a ver todos los días. Esta situación ayudó a que uno se pueda dar cuenta de las verdaderas amistades que uno tiene.

Por otro lado, hay personas que tuvieron varios problemas al quedarse dentro de casa con los miembros de su familia ya que estos no están acostumbrados a tener una convivencia frecuente con los mismos, como por ejemplo aprender a hacer los quehaceres de la casa, ayudar a cocinar, que cada uno ordene como debe su cuarto, entre otros quehaceres que una persona no lo hacía antes.

Se evaluó la confiabilidad de este estudio mediante el Alfa de Cronbach, la cual obtuvo un índice del 0.88, garantizando la certeza de las preguntas y sus resultados.

Este artículo está compuesto por cinco secciones, introducción que es la primera, la que nos describe el problema a tratar. Desarrollo, donde se detallan los aspectos teóricos y argumentos que sustentan nuestra investigación. Se describe la metodología utilizada para el estudio y la obtención de los datos necesarios. Por último, se muestran los resultados del análisis de datos y las conclusiones que estos generan.

\section{II.DESARROLLO}

Se puede observar claramente que ningún país en el mundo está preparado para este tipo de amenazas lo afirma Stefanoni [1]. Al hablar de la terminología pandemia, se hace referencia a una enfermedad de carácter epidemiológico el cual, posee la facultad de extenderse alrededor de la mayoría de las naciones del globo terráqueo, es decir, afecta a sus individuos de forma directa [4]. Esto puede tener una repercusión en términos exponenciales en las interacciones humanas, debido a que genera una amenaza tanto económica como física. Esta emergencia médica orilló a la humanidad a modificar las costumbres y tradiciones de la convivencia cotidiana, ya que el contacto físico entre individuos incrementa la probabilidad de contagio entre las personas como una cadena o red de distribución epidemiológica. Por ello, se debe precautelar la integridad de la sociedad mediante el confinamiento de las personas y limitarse a realizar actividades exteriores en lo mayor posible. De esta manera se logrará vencer o por lo menos encapsular esta tragedia mundial lo cual afecta a cada una de las naciones como a las personas tanto física, económica como socialmente.

Existen diferentes teorías con respecto a la creación como también de la propagación de este virus, las cuales aún no se pueden validar en su totalidad. Lo que se puede afirmar hasta la actualidad es su presunta propagación, la cual se estima que se efectuó a finales del año 2019 en la República China específicamente en la ciudad de Wuhan. La notificación de este virus se realizó el día 31 de diciembre del 2019 por medio de la Organización Mundial De la Salud (OMS), el cuál fue llamado genéricamente como COVID-19 por su caracterización científica como "CoV” y el año de su presunta aparición. El COVID-19 o mejor conocido como "Coronavirus" hace referencia a una extensa familia de virus los cuáles tienen una amplia gama de afecciones empezando desde un resfriado hasta caracteres más complejos que provocan la muerte de los individuos contagiados. Por ello su detección tiene un gran nivel de dificultad debido a que según su cepa puede generar diferentes tipos de reacciones de carácter respiratorio. Es decir, al hablar de las cepas básicamente se hace referencia al tipo de síndrome o afección por síntomas que el paciente muestra. Existen dos tipos de cepas hasta la actualidad y cada una de ellas tiene sus subdivisiones. Una de ellas es el "MERS-CoV" el cuál se sitúa generalmente en Oriente medio por otro lado nos encontramos con otra cepa la cuál 
es denominada como "SRAS-Cov" cuya situación tiene una dimensión más amplia alrededor de las naciones [2].

Con esta nueva normalidad debemos regirnos a grandes cambios, cada país tuvo su manera de enfrentar esta situación con rigurosas medidas de prevención a las que todos los ciudadanos tuvimos que adaptarnos y con esto lograr reducir la tasa de contagios. Lo que se analizó fue la frecuencia con la cual las personas salían de sus casas y sus motivos para ello. Las preguntas de nuestra investigación que nos generaron mayor interés fueron:

- Centros comerciales: Son lugares bastante concurridos por su variedad de tiendas y la utilidad que estas presentan al público además del ocio.

-Hospitales: Lugar que utilizamos para curar cualquier enfermedad o recibir una asistencia médica por alguna emergencia

-Visita a familia: Es bastante común que una familia no viva en un mismo hogar, lo cual hace que el visitar a un familiar, amigo o ser querido sea una actividad bastante frecuente y una razón común para salir de casa.

- Gimnasio: Establecimiento el cual nos facilita el entrenamiento y bienestar de nuestra salud, fomentando las conglomeraciones de gente y el contacto físico.

- Viajar a otras ciudades o países: La frecuencia con la que uno sale del país o cuidad, puede verse altamente afectada por las restricciones del país o cuidad de destino.

- Nivel de protección: Impedimento de que el virus se propague con mayor facilidad.

Los puntos desarrollados anteriormente fueron en los que se basaron las preguntas del estudio, pues se consideran lugares de mucha concurrencia que aumentan la frecuencia con la que la gente decide salir de casa. En la actualidad, salir de casa puede ser considerado hasta una necesidad, para trabajar, socializar, comprar, reabastecerse, estudiar, entre otros.

María Báguena [3] afirma que a lo largo de la historia han existido varias pandemias por diversas enfermedades como: la peste negra, la gripe española, la colera, peste bubónica, u otras; en estas pandemias pasadas, la información de la gente era bastante limitado por lo cual primaba el miedo y descontrol social. Ahora es mucho más sencillo comunicar a la gente las medidas correctas de prevención, teniendo como la más importante el distanciamiento social. Por lo cual se entiende que debería haber una disminución en la frecuencia con la que salía la gente y sus hábitos sociales.

\section{III.METODOLOGÍA}

En este estudio, se utilizó como metodología una encuesta de veintisiete preguntas que tenían un rango de respuesta del uno al cinco, siendo uno muy poco frecuente y cinco con mucha frecuencia. La encuesta pretendía ver la diferencia entre los hábitos actuales en contraste con los hábitos antes de la pandemia, por lo cual las veintisiete preguntas se dividen en catorce preguntas para el antes y trece preguntas para el después. Esto se lo realizó y tabuló de forma virtual mediante la aplicación de Google Forms.

La encuesta estuvo dirigida 20-60 años y todos de nacionalidad ecuatoriana que están bajo las mismas restricciones gubernamentales.

La forma de validación de las preguntas en la encuesta fue mediante el coeficiente del alfa de Cronbach; obteniendo un valor de 0.88 , lo cual indica que las preguntas son estadísticamente validas y sus resultados son certeros. La siguiente formula pertenece al coeficiente de evaluación utilizado:

$$
\propto=\frac{K}{K-1}\left(\frac{\sum_{i=1}^{K} \sigma_{Y i}^{2}}{\sigma_{X}^{2}}\right)
$$

\section{IV.RESULTADOS}

Del estudio realizado se pudieron obtener los siguientes resultados: 
Tabla I. Frecuencia de salida y su motivo

\begin{tabular}{|c|r|r|}
\hline \multirow{2}{*}{ Preguntas } & \multicolumn{2}{|c|}{ Respuesta promedio } \\
\cline { 2 - 4 } & ANTES & DESPUES \\
\hline Al supermercado & 3 & 2 \\
\hline Centros Comerciales & 4 & 1 \\
\hline Hospitales & 2 & 1 \\
\hline Visita a la familia & 4 & 1 \\
\hline Ir a un lugar de entretenimiento & 3 & 1 \\
\hline Lugar de belleza & 3 & 2 \\
\hline Gimnasio & 3 & 1 \\
\hline Parque & 3 & 1 \\
\hline Deporte al aire libre & 3 & 2 \\
\hline Viajar a otra cuidad o país & 3 & 2 \\
\hline Salida de miembros del hogar & 4 & 2 \\
\hline Nivel de protección & 3 & 4 \\
\hline Farmacias & 3 & 2 \\
\hline
\end{tabular}

La Tabla I muestra las respuestas promedio de cada pregunta clasificándolas en su antes de la pandemia y su después para una sencilla comparación visual. La información de la tabla se interpreta de la siguiente manera: el texto del lado derecho indica un lugar a la que una persona puede salir y el número en su fila indica su frecuencia, evaluada del 1 al 5, de salir de casa por dicha razón antes y después de las medidas sanitarias de la pandemia. Cabe destacar dos excepciones: el enunciado de 'Nivel de protección' referencia a el cuidado y protección personal que una persona usaba al salir de casa, evaluada del 1 al 5; 'Salida de miembros del hogar' indica la frecuencia con la que los miembros del hogar salen de casa.

Como se puede observar, la respuesta promedio correspondiente al tiempo del antes de la pandemia, lleva números mayores a los que corresponden al tiempo de después de la pandemia por coronavirus. Esto quiere decir, que antes la gente tenía una mayor frecuencia al salir por los motivos mencionados en la Tabla I. Los resultados son bastante lógicos, pues antes de la emergencia sanitaria no había restricción alguna para la interacción social. El hecho de que después de la pandemia se instalasen restricciones gubernamentales, limitados horarios de salida, la necesidad de salvoconductos para salir, entre otras prohibiciones y controles limita de forma radical el comportamiento humano, lo que explica perfectamente los resultados obtenidos y su disminución notable en la frecuencia promedio de salida.

Cabe recalcar, que la única pregunta que aumento su índice del antes al después es la del nivel de protección. En esta pregunta se consultaba acerca del nivel de protección personal que uno utilizaba al momento de salir. Esta pregunta era la única cuyo valor de frecuencia se esperaba que aumentara, pues es bastante lógico que la gente en pandemia tenga una mayor preocupación por su protección e higiene al momento de salir. Esto a consecuencia de toda la información que se ha dado a la población acerca de la importancia y obligación del cuidado personal para evitar ser un punto de contagio.

\section{V.CONCLUSIONES}

La vida en general tanto nacional como internacional ha dado una vuelta de 360 grados, al cambiar las costumbres que se tenían. El uso de mascarilla como así también la restricción de movilidad son dos de los aspectos más importantes a los cuáles se ha expuesta la sociedad. Dicho esto, se subdividen diferentes restricciones a las cuales nos exponemos hoy en día lo que hablando en términos se está convirtiendo en una necesidad.

Como se mencionó anteriormente con los resultados obtenidos gracias a la aplicación de la estadística podemos identificar puntos críticos e importantes en este análisis, se ha podido comprobar que existe una disminución total con las actividades habituales las que se tenían antes de la pandemia. Sin embargo, al analizar minuciosamente los resultados en la gráfica podemos ver que existe una anomalía ideológica con respecto al flujo de personas hacia farmacias. Ya que se supone y se asume que la mayoría de las personas, evitan acudir a sitios de alta probabilidad 
de consumo masivo, lo cual, nos dirigiría tanto a hospitales como farmacias. Sin embargo, al parecer las personas constantemente están acudiendo por ello.

Otro parámetro el cuál es muy relevante estudiarlo y se relaciona directamente con lo anteriormente expuesto y generaría una justificación. Es el uso e implementación de protección personal para combatir esta pandemia. Por ello, asumimos que existe flujo de personas en farmacias debido al constante abastecimiento de estos insumos.

Al hablar de la constante importancia que se está dando por la sociedad, se puede asumir y dictaminar una hipótesis, de que se va a generar un cambio de ideología en temas tanto de limpieza como salubridad en los seres humanos. Lo cual, nos daría un indicador que se va a modificar positivamente las culturas y tradiciones de nuestro país con respecto al aseo e higiene en las personas.

Nuestra sociedad está teniendo un cambio radical positivamente ya que se va a valorar y respetar más el entorno en el que se convive para que se pueda prevenir cualquier tipo de desastre en la humanidad.

\section{REFERENCIAS}

[1]P. Byass, «Eco-epidemiological assessment of the COVID-19 epidemic in China, January-February 2020,» Web Of Science, vol. 13, $\mathrm{n}^{\circ} 1,2020$.

[2]P. Stefanoni, «Brasil: pandemia, guerra cultural y precariedad,» Nueva Sociedad, pp. 49-59, 2020.

[3]M. J. Báguena Cervellera, «La pandemia de COVID-19 a la luz de la historia de la medicina,» Investigación y Ciencia , 2020.

[4]A. Levy, «La pandemia de COVID-19 podr\{ia ayudar a resolver una gran incógnita climática,» Investigación y Ciencia, 2020.

[5]T. Marcel Ariel, «Relaciones en tiempos de pandemia: COVID-19 y bienestar animal, ambiental y humano,» Revista Facultad Nacional De Agronomia Medellin, vol. 2, 2020.

[6]F. Manrique-Abril, «Modelo SIR de la pandemia de Covid-19 en Colombia/SIR model of the COVID-19 pandemic in Colombia,» Revista De Salúd Publica, vol. 22, pp. 1-6, 2020.

[7]D. Arango-Londoño, "Predicciones de un modelo SEIR para casos de COVID-19 en Cali, Colombia/Predictions of a SEIR model for COVID-19 cases in Cali-Colombia,» Revista De Salúd Publica, vol. 22, n 2, pp. 1-9, 2020. [8]J. Gonzales-Castillo , «Pandemia de la COVID-19 y las Políticas de Salud Pública en el Perú: marzo-mayo 2020/ COVID-19 pandemic and Public Health Policies in Peru: March-May 2020,» Revista De Salúd Publica, vol. 22, nº 2, pp. 1-9, 2020.

[9]A. Valenzuela-Cazés y L. Becerra-Ostos, «Práctica clínica, ámbito laboral y riesgos de la fisioterapia ante el COVID-19/Clinical practice, work and risks of physical therapy in the face of COVID-19,» Revista De Salúd Publica, vol. 22, no 2, pp. 1-4, 2020.

[10]P. Montes-Alarcón y A. Campo-Arias, «Los médicos generales y la salud mental en la pandemia por COVID-19,» Duazary, vol. 17, no 3, pp. 4-6, 2020.

\section{RESUMEN CURRICULAR}

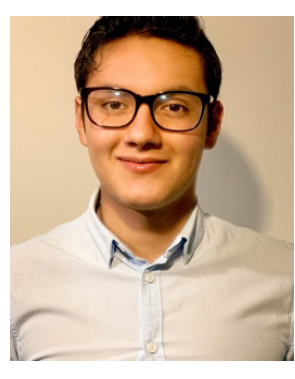

Marcelo Nicolás Salazar Roldán, ecuatoriano nacido el 03 de diciembre de 1999, en la ciudad de Quito Ecuador. Actualmente se encuentra desempeñando sus labores profesionales en el área de producción en la empresa Dimabru Cia. Ltda. Estudiante de la carrera de Ingeniería Industrial en la Universidad de las Américas. Entre sus principios están el respeto, transparencia, empatía y puntualidad 


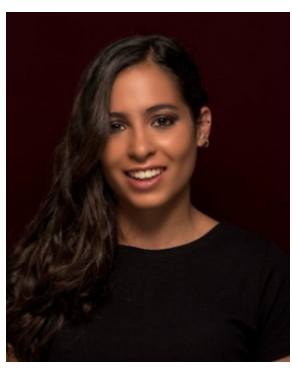

Carmen Santander, Estudiante de Ingeniería Industrial de la Universidad de las Américas. Nacida el 29 de febrero del año 2000 en Quito, Ecuador. Le gustan muchos deportes y esto le ayuda a la concentración para su carrera, se considera una persona sincera ya que siempre habla con la verdad

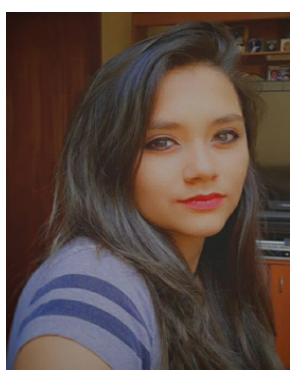

Andrea Micaela Egas López, Estudiante de Ingeniería Industrial de la Universidad de las Américas. Nacida el 7 de marzo del año 2000 en Quito, Ecuador. Le gusta realizar deporte con su familia, y también realizar manualidades que sean de concentración

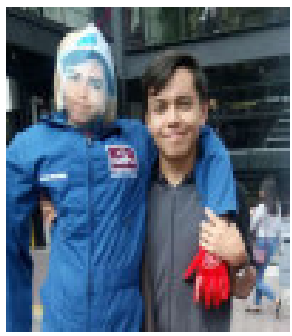

Alejandro Sebastián Grijalva Mora, estudiante de Ingeniería Industrial en la Universidad de las Américas. Nacido el 5 mayo del año 2000 en Quito, Ecuador. Aficionado por las ciencias exactas como las matemáticas y la física 